\title{
DECAYED WOODY MATERIAL FROM MUSHROOM CULTIVATION: CHARACTERIZATION OF LIQUEFACTION
}

\author{
QINGYUE WANG \& SAYURI KAWAMURA \\ Graduate School of Science and Engineering, Saitama University, Japan
}

\begin{abstract}
Decayed woody material from mushroom cultivation is one of the most important waste materials in Japan, as almost 300 tonnes are released each year. It is nearly impossible to reuse these resources, because of the damage to its original form and deterioration of its strength. These decayed woody materials could be used to create a valid chemical product from the main components' decomposition: this could reduce waste material and its environmental impact, especially in developing countries. This article focuses on the liquefied portion of decayed woody materials that is released from mushroom cultivation. By analysis of the products from major woody holocellulose (i.e. cellulose and hemicellulose) and lignin, we found the same ratios of weight loss in decayed woody samples, compared with the original woody samples. All the main components of the decayed wood were partially degraded. The crystallinities within woody samples were decreased with sufficient long-term decay. After these decayed woody samples were liquefied and compared with the original samples, we conducted liquefaction tests using ethylene glycol, glycerine, and the catalyst sulfuric acid. The ratios of residue during liquefaction showed that the liquefied characteristics of decayed woody material released from mushroom cultivation were equal to or less than that of the original wood. In addition, the results as determined by a Fourier-transform infrared spectroscopy showed that the liquefied ratios of decayed woody samples could be equal to or higher than those original woody samples without decay.
\end{abstract}

Keywords: agricultural waste, decayed wood, liquefaction, mushroom cultivation, white-rot fungus, wood decay, forestry waste.

\section{INTRODUCTION}

There is no doubt that these days the world has many environmental problems, such as the escalation of $\mathrm{CO}_{2}$ levels, desert expansion, soil erosion, elevated temperatures, extinction of certain species, falling water tables, melting glaciers, grassland deterioration, dried-up rivers, drought, floods, and more. These environmental problems not only affect developing countries, but also developed countries, where they hinder economic development. What we extract from the earth for basic needs and economic development has been greater than that which the Earth can replenish by itself; thus, economic development has always been accompanied by the out-stripping of the Earth's natural resources. Environmental problems could be a possible cause of more poverty and competition among consumers. In particular, people living in developing countries use local resources from nature for their daily lives, and their population is expanding rapidly: they are using up all the resources before these can be restored to their former condition. If competition breaks out for the dwindling natural resources, then these could become further destroyed and the amount of waste could increase. It is commonly known as a vicious cycle, when people destroy the natural environment due to poverty; which may cause war among the people and, in turn, make them poorer.

To solve those problems, it is necessary to build a sustainable society. Sustainable development must be the organizing principle for meeting human development goals, while at the same time, sustaining the ability of natural systems to provide the natural resources and ecosystem services upon which the economy and society depend. Therefore, we must 
think of a valid way to mitigate the present crisis, such as the proper utilization of waste materials, converting them into natural resources.

At present, most wood decays after being cultivated, becoming waste materials, with little possibility of reusing this resource: 130,000 tons of these waste materials are discharged annually. The mushroom industry is an important one, accounting for $40 \%$ of the forestry industry in Japan [1]. On the other hand, woody biomass, which could contribute to sustainable development by conjugating effectively as sustainable materials and activating the mountain village regions, creating new industry and employment opportunities, has received more attention.

Wood cannot change its shape as easily as metal or plastic; thus, the utility value of the material is very low, because the wood can only be adapted to a limited number of processing methods. However, when liquefied, wood has added value. Wood liquefaction is one of the most effective technologies for biomass utilization [2]. When the woody powder is heated, mixing it with an organic solvent in the presence of an acid catalyst produces a black viscous liquid material. This material is called liquefied wood; and, with the addition of chemical reagents, can be made to form a resin [3]-[5].

The world is faced with energy problems in terms of the depletion of crucial reserves of fossil fuels. Liquefaction is one of the technologies for producing resin, which has traditionally been produced from petroleum, but with the advantage of saving petroleum resources; therefore, a higher rate of biomass is preferred. The effect of condensation is known to impede a higher rate of biomass in liquefied wood [6]-[8]. Pre-treatment is used to inhibit condensation [9]-[11]. In this way, liquefaction involves decomposing the main component using a solvent and catalyst, and the level of decay is much higher in the same process, when occurring through wood-rot fungus. This means that the decay could be considered a pre-treatment process. The woody samples which decayed by means of brownrot fungus (one of the three categories of wood-rot fungi) were liquefied better than the original woody samples, because of a relatively large amount of lignin following the decomposition of cellulose and the destruction of the crystallinities of cellulose [12]. Additionally, in previous work, the woody samples were decayed by white-rot fungus, and weight loss and morphologic changes were measured and used to experiment with liquefaction, which indicated that the decayed wood could become liquefied in the same way as non-decayed wood [13]. Since there was less information about white-rot fungus and the decayed woody materials from mushroom cultivation, this study paid greater attention to conducting the liquefied experiment with them and analysing the components.

\section{MATERIAL AND METHODS}

\subsection{Preparation of woody samples}

An original cherry tree (sakura tree) and decayed woody materials from mushroom cultivation from Yamaguchi Shintan, Yamagata, Japan, were prepared. These woody samples were crushed. The pulverized wood was classified to two groups. One was over $250 \mu \mathrm{m}$, the other less than $250 \mu \mathrm{m}$. The woody powder samples which were less than $250 \mu \mathrm{m}$ were dried at $105^{\circ} \mathrm{C}$ overnight, then their weights were measured.

\subsection{Analysis of components of woody samples}

The samples were defatted using soxhlet extraction. In total, $50 \mathrm{~mL}$ of ethanol and $100 \mathrm{~mL}$ of benzene were used to make the defatted woody samples [6]. 
To analyse the content ratio of holocellulose, we conducted examinations using the following method: $2.5 \mathrm{~g}$ of the defatted woody samples were put in a $300-\mathrm{mL}$ flask with 150 $\mathrm{mL}$ of pure water, $1.0 \mathrm{~g}$ of sodium chlorite and $0.20 \mathrm{~mL}$ of acetic acid, then heated at $75^{\circ} \mathrm{C}$ in a water bath, with occasional stirring; $1.0 \mathrm{~g}$ of sodium chlorite and $0.2 \mathrm{~mL}$ of acetic acid were added three times at four-hour intervals; then these contents were filtered, using a glass filter (1GP100, AS ONE Co. Ltd., Japan), and rinsed with pure water and acetone. The residual solid left was holocellulose.

Analysis of the content ratio of lignin was performed by the Klason lignin method. We placed some $1.0 \mathrm{~g}$ of the defatted wood sample and $15 \mathrm{~mL}$ of the $72 \%$ sulfuric acid in a $50 \mathrm{~mL}$ beaker and mixed, with occasional stirring, for four hours. These contents were poured into a $1 \mathrm{~L}$ Erlenmeyer flask, which was filled with $560 \mathrm{~mL}$ of extra-pure water. This flask was fitted with a condenser and heated at $110^{\circ} \mathrm{C}$ in an oil bath for four hours. These contents were then filtered, using a glass filter (1GP16, AS ONE Co. Ltd., Japan), and rinsed with hot water and pure water. The residual solid was lignin.

\subsection{Measurement of the crystallinity of woody samples using X-ray diffraction}

Cellulose is known to have a crystal-like structure. Degradation by wood-rot fungus could decrease the crystallinity of cellulose. Therefore, the crystallinity of the woody samples was measured by X-ray diffraction (XRD) (Rint UltimaIII, Rigaku Corporation, Japan). The holocellulose of the woody samples was used for the XRD analysis. Additionally, a baseline was set between the intensity values of $10^{\circ}$ and $30^{\circ}$ of the diffraction angle, and the crystallinity was calculated as shown in eqn (1), as the area above the baseline was the crystalline region and the area beneath the baseline was the amorphous region, $10^{\circ}$ and $30^{\circ}$ of the diffraction angle

$$
\text { crystallinity }(\%)=\frac{s_{c}}{s_{c}+S_{a}} \times 100
$$

where $S_{c}$ : the area of the crystal region; $S_{a}$ : the area of the amorphous region.

\subsection{Liquefaction of woody samples}

In a $500 \mathrm{~mL}$ three-neck reaction flask equipped with a stirrer and a reflux condenser [5], we measured $10 \mathrm{~g}$ of the woody samples and $60 \mathrm{~g}$ of the mixed solution (glycerine: polyethylene glycol = 1:9) and mixed. Then, the reaction flask was immersed in a preheated oil bath at three different temperatures: $120^{\circ} \mathrm{C}, 130^{\circ} \mathrm{C}$, and $140^{\circ} \mathrm{C}$. The reaction started when $0.1 \mathrm{~g}$ of the catalyst $\left(\mathrm{H}_{2} \mathrm{SO}_{4}\right)$ was added to the reaction flask after 10 minutes of premixing. After liquefaction reaction times of 15, 30, 90, 180 and 300 minutes; the liquefied woody samples were collected and placed into a weighted vial, in order to analyse the residue contents. The vial was immersed in cold water to stop the liquefaction reaction. To filter the residue, $10 \mathrm{~mL}$ of methanol was added to the vial and combined completely, using a shaking apparatus. Filtering was performed by using a pump (EVP-1000, KNF Co. Ltd) and filters (C5, ADVANTEC). The filtered residue was dried for 24 hours in an oven at $105^{\circ} \mathrm{C}$. The rate of residue was calculated as shown in eqn (2)

$$
\text { ratio of residue }(\%)=W_{1} / W_{0} \times 100 \text {, }
$$

where $\mathrm{W}_{1}$ : weight ratio of residue in the taken liquefied sample; $\mathrm{W}_{0}$ : weight ratio of wood in the initial sample. 
2.5 Measurement of functional groups of liquefied woody samples by FT-IR

To check liquefaction, the functional groups of liquefied woody samples were measured by Fourier-transform infrared spectroscopy (FT-IR) (JASCO Co. Ltd., Japan). The liquefied woody sample was clipped thinly by potassium bromide plates. The pellet was set in FT-IR, and functional groups of liquefied woody samples were measured.

\subsection{Molecular weight distribution}

To analyse the change in molecular weight distribution by reaction time, the molecular weight distribution of liquefied woody samples was measured by gel permeation chromatography (GPC). First, 10-ml samples were dissolved in $10 \mathrm{ml}$ tetrahydrofuran (THF) $(0.1 \mathrm{w} / \mathrm{v} \%)$ and then filtered through a $0.45 \mu \mathrm{m}$ syringe filter. Then, samples were injected into a column (KF-802, SHODEX Co. Ltd., Japan), and placed in a high performance liquid chromatography pump (HPLC Pump PU-2080, JASCO Co. Ltd., Japan), and a refractive index-detector (RID) (RI-2031, JASCO Co. Ltd., Japan). The measurement was performed at $40^{\circ} \mathrm{C}$ for $10 \mathrm{~min}$, with $1 \mathrm{ml} / \mathrm{min}$ of $\mathrm{THF}$ as eluent.

\section{RESULTS AND DISCUSSION}

\subsection{Comparison of the condition of the wood}

\subsubsection{Ratio of the ingredients of the wood}

Fig. 1 shows the results of the component analysis. Although there were no obvious differences between the original cherry-blossom tree (sakura tree) (control) and the decayed woody materials from mushroom cultivation (cherry-blossom tree, decayed scrap), the lignin in the decayed woody materials had increased slightly, by $5 \%$, while the holocellulose had decreased.

The level of decomposition of cellulose, hemicellulose and lignin differed according to the type of fungi, wood, and the degree of decay; however, in the case of the wood whose degree of decay was sufficient, they mostly decomposed at the same time, because white-rot

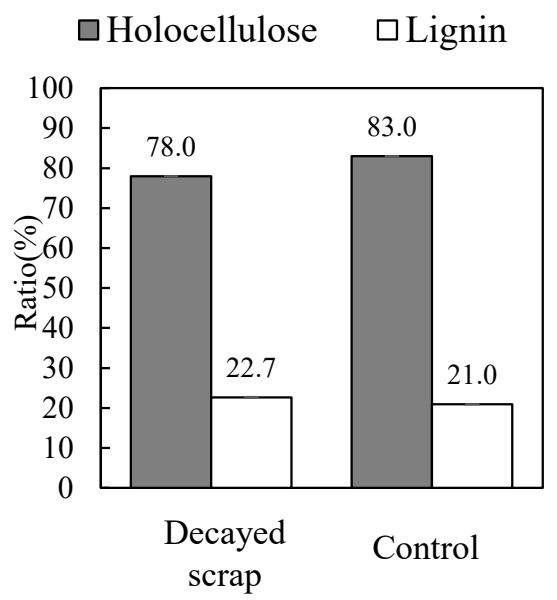

Figure 1: The holocellulose and lignin ratios of wood samples (decayed scrap from mushroom cultivation; and original control woody samples). 
fungus could rot each component at the same time. Similar results were also reported during the examination of spruce decayed by white-rot [14]. In this study, each component also decomposed at the same time, and there were no big differences between samples.

\subsubsection{Crystallinity}

Fig. 2 shows the result of the XRD spectra of the woody samples. The strength of the peak of the decayed scrap demonstrates the devolution of the crystal structure rather than that of the control. Table 1 shows the calculated crystallinity values of the decayed scrap and the control, following eqn (1) from the peak XRD used. In Table 1 we show the crystallinity calculated from the peak.

Howell et al. [15] showed that crystallinity increased at the initial step in a term of decay using brown-decay fungus, when compared with that of another type of decay. Hastrup et al. [16] demonstrated that, in the case of white-rot fungus, the crystallinity was found to increase during some early stages of degradation, the reason being that it decomposed into being noncrystalline first. In this study, the crystallinity decreased in relation to that of the control, due to sufficient long-term decay.

\subsection{Results of liquefaction of woody samples}

\subsubsection{Residue content change during liquefaction}

Fig. 3 shows the residue content as a function of liquefaction time for each liquefaction temperature $\left(120,130\right.$ and $\left.140^{\circ} \mathrm{C}\right)$, respectively. All of these revealed that the residue percentage descended rapidly to about $50 \%$ within 15 minutes of reaction time. This indicated that the lignin and holocellulose were being liquefied. When the next 15 minutes were considered, the residue percentage was still decreasing, but at a slower rate. Degradation

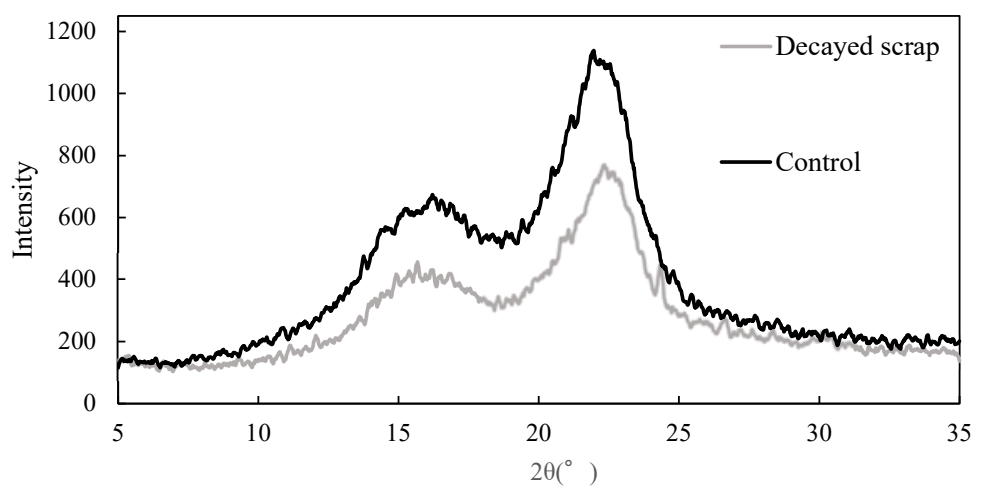

Figure 2: The XRD spectra of the woody samples (decayed scrap from mushroom cultivation and original control woody sample).

Table 1: The crystallinity of the decayed scrap from mushroom cultivation and original control woody samples.

\begin{tabular}{|l|c|}
\hline Woody sample & Crystallinity (\%) \\
\hline Control & 59.3 \\
\hline Decayed scrap & 47.5 \\
\hline
\end{tabular}




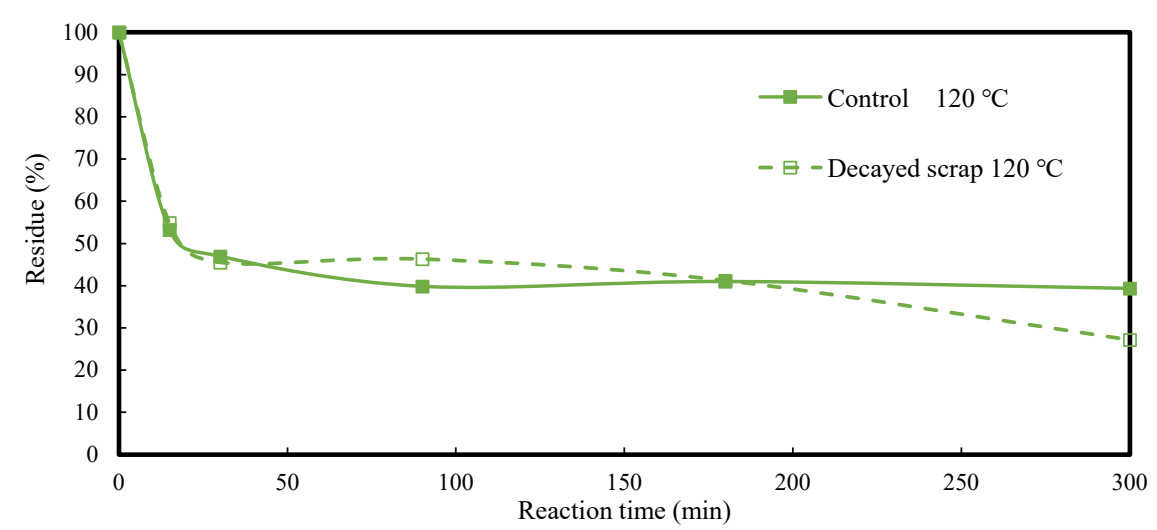

(a)

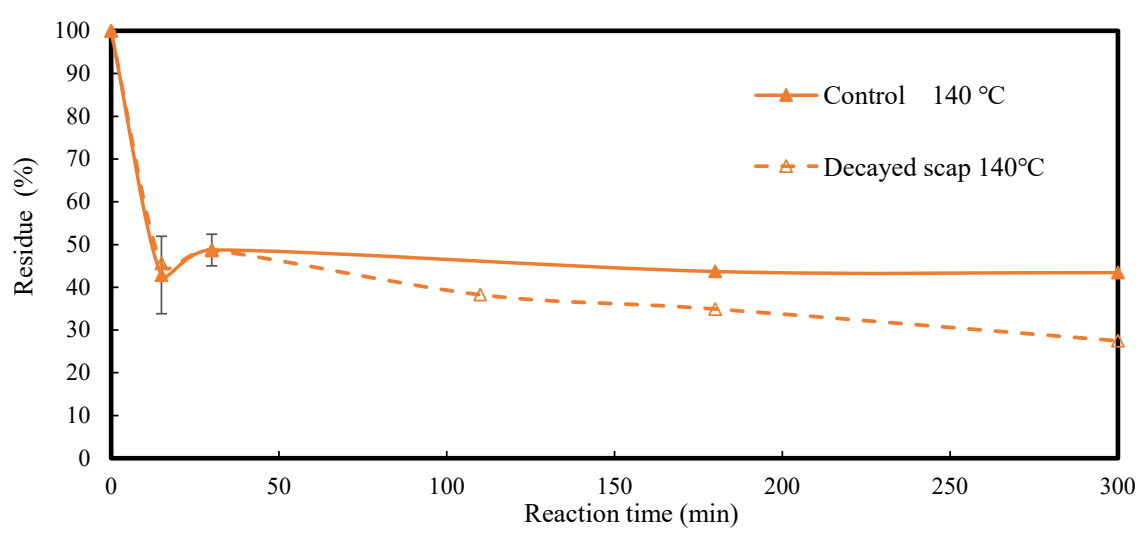

(b)

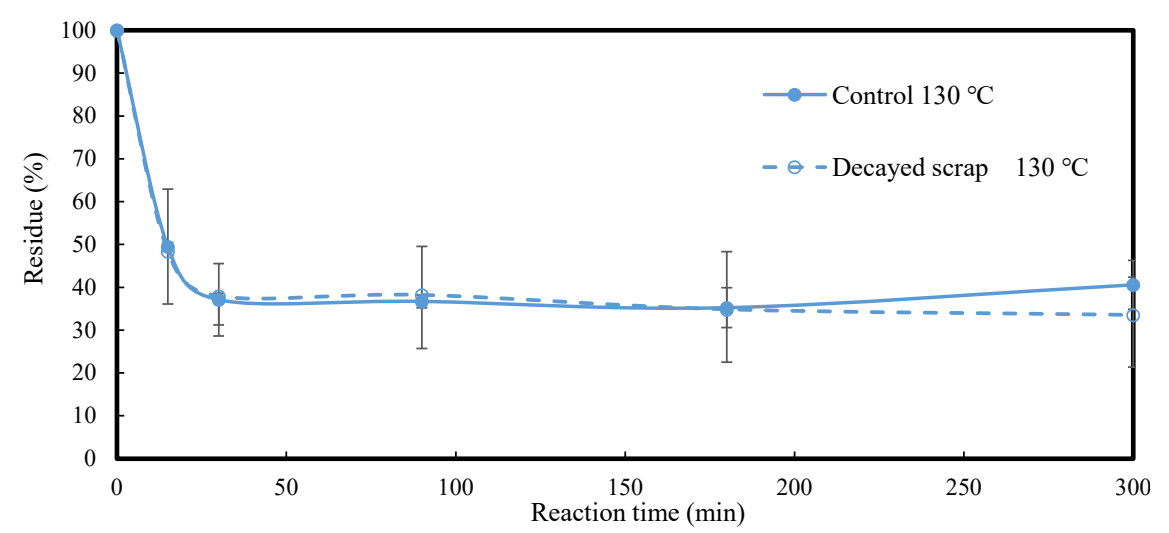

(c)

Figure 3: Reaction of the residue of liquefaction in the woody samples; comparison between decayed scrap from mushroom cultivation and the original control woody sample at (a) $130^{\circ} \mathrm{C}$; (b) $140^{\circ} \mathrm{C}$; and (c) $150^{\circ} \mathrm{C}$. 
makes the woody material decompose and reduced the residue percentage. In contrast, condensation produced insoluble material and increased the residue percentage. At the earlier stage of liquefaction, the effect of degradation is stronger than the effect of condensation, resulting in the residue percentage decreasing rapidly

As shown in Fig. 3, decayed scrap became liquefied to an equal or higher level than the control. The main reason is that the crystallinity of holocellulose was hard to deliquefy. White decay fungus decomposes lignin with cellulose and hemicellulose at the same time. On the other hand, brown decay fungus decomposes only cellulose and hemicellulose and the decomposition occurs more rapidly than absorption [17]. For these reasons, one previous study of white decay fungus showed that lignin did not increase as much as the brown decay fungus, and crystallinity did not decrease, and liquefaction of white decay fungus, of which the ration of residue is the same as control; however, based on previous findings, the ratio of lignin was slightly higher in the decayed scrap, and the crystallinity decreased; the decayed scrap could be liquefied to an equal or greater level than the control.

The influence of the reaction temperature is shown in Fig. 4. In both the control and the decayed scrap, although the lowest rate of residue was obtained at $130^{\circ} \mathrm{C}$ or $140^{\circ} \mathrm{C}$, almost all the data was in the error range of the residue percentage of $130^{\circ} \mathrm{C}$ and the data from 300 minutes showed the same ratio. These results suggested that the reaction temperature caused no difference in residue content in the range of this experiment, and this also applied to the decayed scrap. The liquefaction reaction could be said to be a balance between the degradation and condensation reactions. The degradation reaction is dominant when the temperature is low. It is suggested that an increase in residue content was not shown at low temperature, due to the low reaction temperature. Wang and Chen [18] also said that, when a liquefied temperature is $140^{\circ} \mathrm{C}$ or lower, degradation is more important, and the residue percentage is decreased and liquefied; while at over $150^{\circ} \mathrm{C}$, the concentration of the small molecule becomes high and the condensation reaction takes precedence.

All the data is combined in Fig. 5, which suggests the following: temperature made no difference; all of the residue content was the same at the initial term of reaction. With a longer the reaction time, the residue content of the decayed scrap became less than that of the control. During the process of liquefaction treatment, first the lignin gets decomposed; but meanwhile, denaturation of hemicellulose also proceeds. Furthermore, the polysaccharides of the non-crystalline part get liquefied; and then after 180 minutes, the crystalline-centred part's cellulose liquefied. This is the reason why the residue content of liquefied wood was different, based on the ratio of crystallinity between the decayed wood and the control group.

\subsubsection{Functional group structure of liquefied woody samples}

Fig. 6 shows the results of the functional structure measurements by FT-IR. The absorption band of $830 \mathrm{~cm}^{-1}$ was attributed to the bending of aromatic $\mathrm{C}-\mathrm{H}$ single bonds. The absorption bands of 1510 and $1600 \mathrm{~cm}^{-1}$ were attributed to the aromatic ring of lignin. The absorption bands of 1035,1110 , and $1160 \mathrm{~cm}^{-1}$ were attributed to the $\mathrm{C}-\mathrm{O}$ single bond stretching of cellulose. The major bands representing carbohydrates and lignin can be observed, indicating that both the lignin and the cellulose in the woody material were transferred into the liquid phase. The broad band at about $3600-3000 \mathrm{~cm}^{-1}$ relates to the characteristic stretching vibration of $-\mathrm{OH}$. The absorption bands at $1080 \mathrm{~cm}^{-1}$ and $1041 \mathrm{~cm}^{-1}$ are characteristic of hydroxyl groups and assigned to $\mathrm{C}-\mathrm{O}$ stretching vibrations in PEG400, glycerol, and their derivatives. As the liquefied woody samples are a complicated mixture, it is difficult to characterize the liquefaction products simply by functional group structure. 


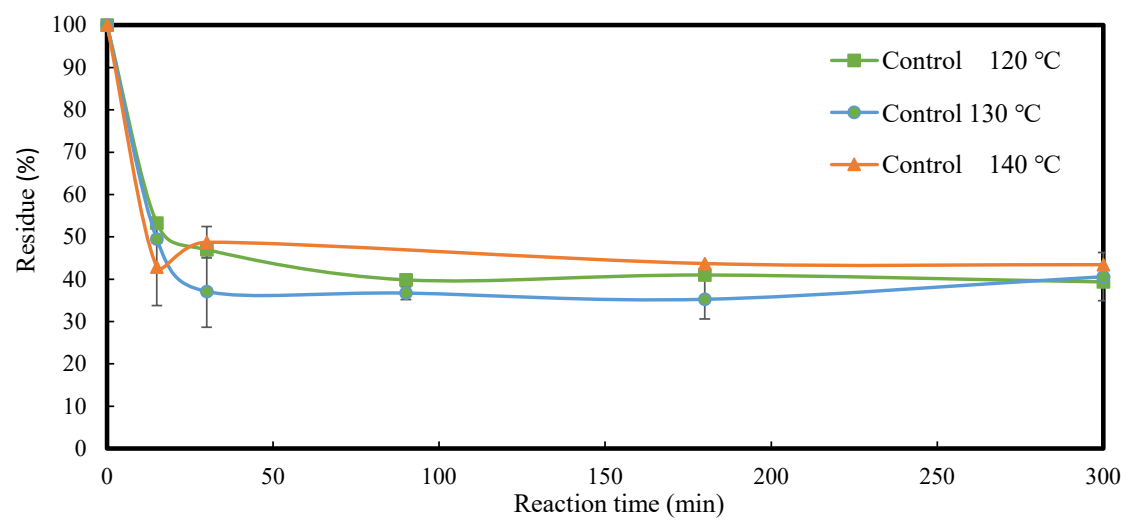

(a)

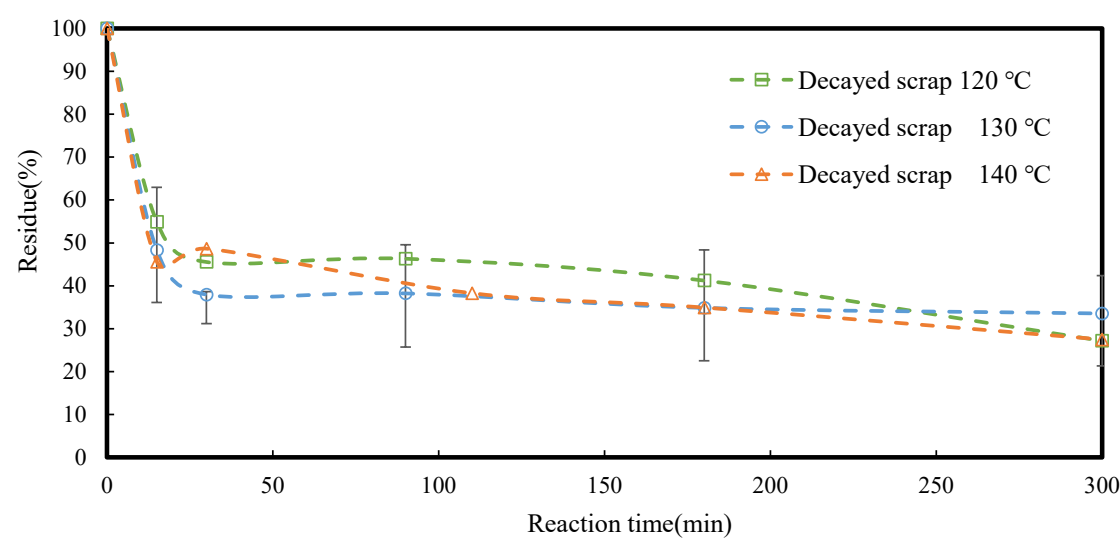

(b)

Figure 4: The residue of liquefaction of the woody samples. Comparison between reaction temperatures. (a) The original control woody sample; and (b) Decayed scrap from mushroom cultivation.

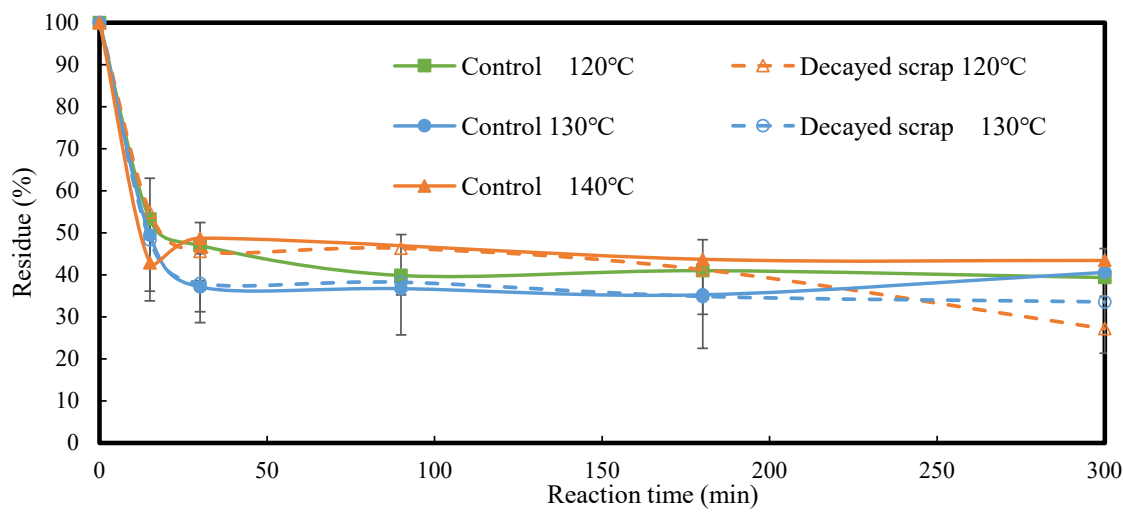

Figure 5: The residue of liquefaction of the woody samples (combining Figs 3 and 4). 


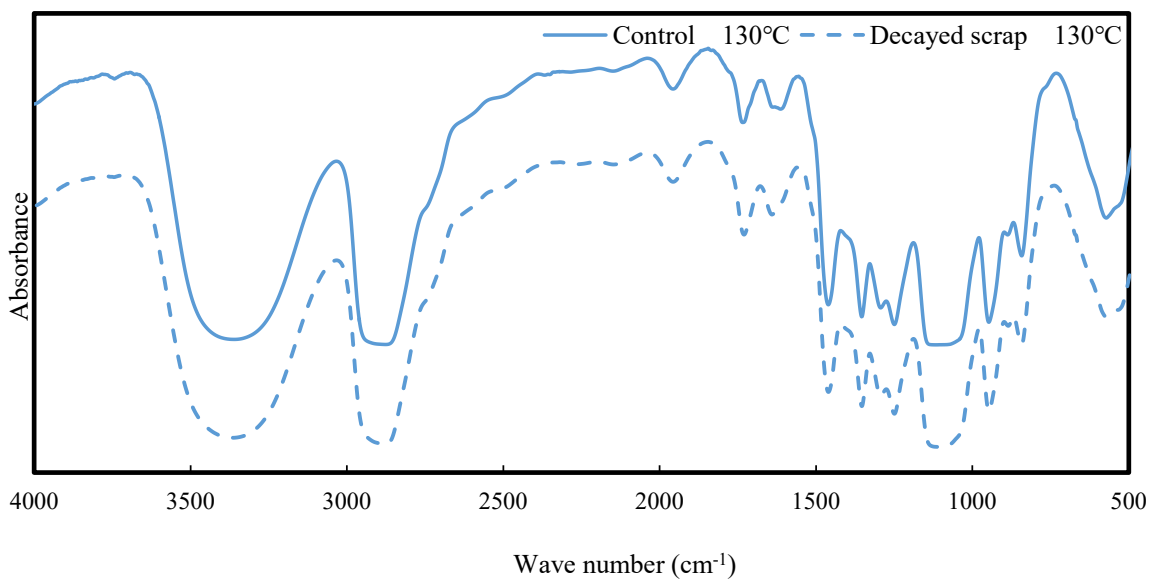

Figure 6: The FT-IR spectra of liquefied woody samples: decayed scrap from mushroom cultivation and original control woody sample (reaction temperature: $130^{\circ} \mathrm{C}$ ).

There was no difference between the decayed scrap and the control wood. Both were decomposed by the liquefaction reaction, as per the above description, and became liquefied wood with the same functional structure. Based on the functional group structure results, it was concluded that the decayed waste material also gets liquefied.

\subsubsection{Measurement of molecular weight distribution}

Fig. 7 shows the results of the measurement of molecular weight distribution of the different reaction times of liquefaction of the decayed scrap (liquefaction temperature $=130^{\circ} \mathrm{C}$. All of the data of the molecular weight distribution represent the peak molecular weight of the high molecular weight fraction and the low molecular weight fraction, respectively. The data show that components with different molecular weights dissolved during the liquefaction procedure. The apparent molecular weight of the liquefied decayed scrap remained relatively constant, irrespective of reaction. This suggests that almost all of the components of the woody sample were liquefied rapidly, within 15 minutes of the beginning of the reaction.

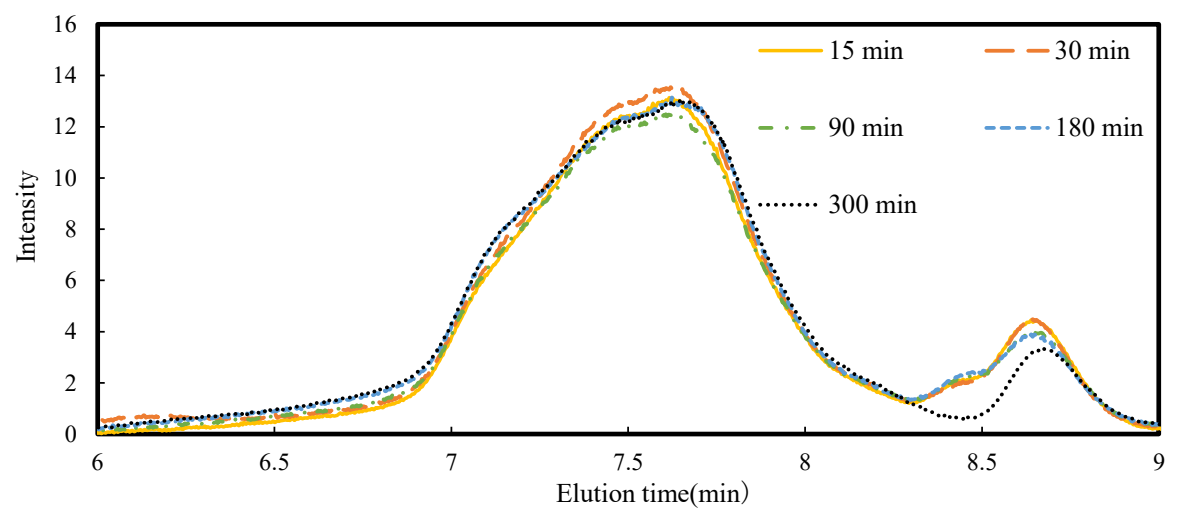

Figure 7: The molecular weight distribution of different reaction times of liquefaction of decayed scrap reaction at $130^{\circ} \mathrm{C}$. 


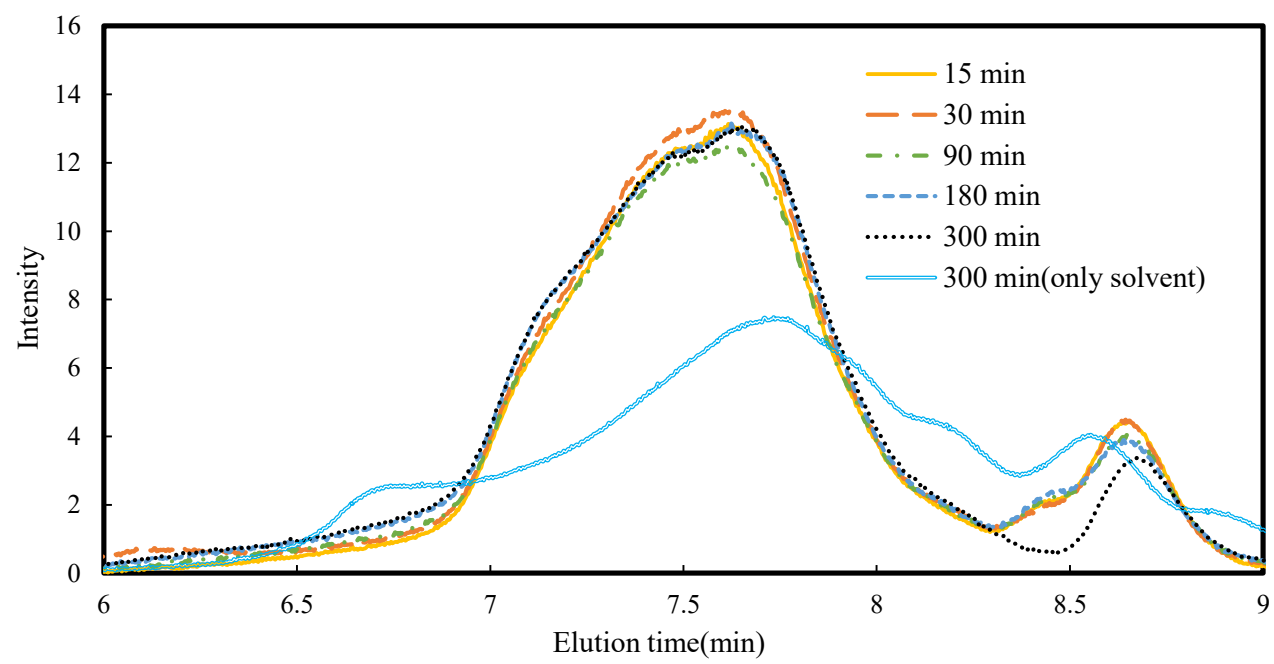

Figure 8: The molecular weight distribution of liquefied woody material and liquefied solvent reaction at $130^{\circ} \mathrm{C}$.

These data indicate that increasing the temperature resulted in a wider molecular weight distribution. This is because increased temperature can promote the simultaneous decomposition of the wood components and the condensation of the liquefaction intermediates.

In this study, based on the results of residue content, there is almost no condensation, due to having had the decay as pre-treatment.

Both the solvent and catalyst were reacted under the condition of $130^{\circ} \mathrm{C}$ for $300 \mathrm{~min}$, which is equivalent with the liquefaction experiment (the only difference is that with no wood). The molecular mass distribution of this reactant and the molecular mass of the liquefied product of decayed wasted materials, as shown in Fig. 8. There are differences between the peak (which show the molecular mass) of reacted solvent and the peak of the liquefied product of decayed waste materials. This suggests that the woody component was dissolved in the liquid, in the case of liquefaction; plus that even decayed scrap could be liquefied.

\section{CONCLUSIONS}

An original cherry-blossom tree (sakura tree) and decayed woody material from mushroom cultivation (also from cherry-blossom tree, but decayed scrap) were used for this study. The content rate of holocellulose and lignin, the main components of wood, were almost the same as in the original control woody sample, when compared with the decayed scrap sample. Decomposition and absorption occurred simultaneously by means of white-decay fungus. In this style of decay, lignin content increased slightly and holocellulose was slightly decreased. Regarding crystallinity, the decayed scrap sample showed lower levels than those of the original control woody sample.

In the examination of liquefied material, the efficiency of the decayed scrap sample was equal to or greater than that of chemical processing of the original control woody sample. The same result in the functional group structure of the liquefied woody sample suggested 
that even the decayed scrap sample can be further liquefied. A molecular weight distribution analysis showed that the woody components were dissolved in the liquid.

According to these results, decayed woody samples can be converted to more valuable material than the original wood, solving the forestry waste problem and improving the environmental problem.

\section{ACKNOWLEDGEMENT}

Some of this work was supported by receiving the Special Funds for Basic Research (B) (no. 15H05119, FY2015 FY2017) from the Grant-in-Aid Scientific Research of the Japanese Ministry of Education, Culture, Sports, Science and Technology (MEXT).

\section{REFERENCES}

[1] Forestry Agency Ministry of Agriculture, Forestry and Fisheries of Japan, Annual Report on Forest and Forestry in Japan, Fiscal Year 2016, pp. 14-29, 2016.

[2] Shiraishi, N., Onodera, S., Ohtani, M. \& Masumoto, T., Dissolution of etherified or esterified wood into polyhydric alcohols or bisphenol A and their application in preparing wooden polymeric materials. Mokuzai Gakkaishi, 31, pp. 418-420, 1985.

[3] Yan, Y., Pang, H., Yang, X., Zhang, R. \& Liao, B., Preparation and characterization of water-blown polyurethane foams from liquefied cornstalk polyol. Applied Polymer Science, 110, pp. 1099-1111, 2008.

[4] Lee, S.H., Teramoto, Y. \& Shiraishi, N., Resol-type phenolic resin from liquefied phenolated wood and its application to phenolic foam. Journal of Applied Polymer Science, 84, p. 468, 2001.

[5] Wang, Q. et al., Liquefaction processes and characterization of liquefied products from waste woody materials in different acidic catalysts. The Sustainable World, Ecology and the Environment, 142, pp. 343-354, 2010.

[6] Wang, Q. et al., Investigation of condensation reaction during phenol liquefaction of waste woody materials. International Journal of Sustainable Development and Planning, 9, pp. 658-668, 2014.

[7] Wang, Q. et al., Suppression method of the condensation reaction during phenol liquefaction of woody material. WIT Transactions on Ecology and the Environment, vol. 176, WIT Press: Southampton and Boston, 2013.

[8] Wang, Q., Effect of the phenol concentrations on the condensation reaction during the liquefaction of waste woody materials with phenol. Waste Management and the Environment VI, Ecology and The Environment, 163, pp. 355-366, 2012.

[9] Wang, Q., Chen, Q., Mitsumura, N. \& Niida, H., Improved cellulose by ionic liquidwater mixture with solid acid catalysis and its application in polyethylene glycol liquefaction. Materials Sciences and Applications, 5, pp. 183-192, 2014.

[10] Chen, Q. \& Wang, Q., Application of ionic liquid and water mixture in cellulose solvent liquefaction as pretreatment step. Biomass Chemical Engineering, 48, pp. 18, 2014.

[11] Wang, Q., Chen, Q., Mitsumura, N. \& Animesh, S., Behavior of cellulose liquefaction after pretreatment using ionic liquids with water mixtures. Journal of Applied Polymer Science, 131, pp. 1-8, 2013.

[12] Li, G.Y., Hse, C.Y. \& Qin, T.F., Preparation and characterization of novolak phenol formaldehyde resin from liquefied brown-rotted wood. Journal of Applied Polymer Science, 125, pp. 3142-3147, 2012.

[13] Wang, Q., Takahashi, H., Kawamura, S. \& Hatakeyama, S., Basic study on decayed behavior of waste woody samples caused by three white-rot fungi. WIT Transactions 
on Ecology and the Environment, vol. 224, WIT Press: Southampton and Boston, pp. 267-277, 2017.

[14] Kirk, T.K. \& Highley, T.L., Quantitative changes in structural components of conifer woods during decay by white- and brown-rot fungi. Phytopathology, 63, pp. 13381342, 1973.

[15] Howell, C., Christine, A., Hastrup, S., Goodell, B. \& Jellison, J., Temporal changes in wood crystalline cellulose during degradation by brown rot fungi. International Biodeterioration \& Biodegradation, 63, pp. 414-419, 2009.

[16] Hastrup, A.C.S., Howell, C., Larsen, F.H., Sathitsuksanoh, N., Goodell, B. \& Jellison, J., Differences in crystalline cellulose modification due to degradation by brown and white rot fungi. Fungal Biology, 116, pp. 1052-1063, 2012.

[17] Takahashi, M., Fungal decay types, their significance in wood preservation. Wood Research Review, 22, pp. 19-36, 1986.

[18] Wang, H. \& Chen, H., A novel method of utilizing the biomass resource: Rapid liquefaction of wheat straw and preparation of biodegradable polyurethane foam (PUF). Chinese Institute of Chemical Engineers, 38, pp. 95-102, 2007. 